\title{
Are generalized call-spreads efficient?
}

\author{
G. Carlier, R.-A. Dana *
}

\begin{abstract}
In order to explain coexistence of a deductible for low values of the loss and an upper limit for high values of the loss in insurance contracts, we consider the exchange of risk between two rank dependent expected utility maximizers. It is shown that if the insurer (insured) takes more into account the lowest outcomes -hence maximal losses- than the insured (insurer), then the optimal contract has an upper limit (includes a deductible for high values of the loss). If furthermore, the insured (insurer) neglects the highest outcomes while the insurer (insured) does not, the optimal contract includes a deductible (full insurance) for low values of the loss.
\end{abstract}

\section{Introduction}

Since Arrow's 1963 seminal insurance paper (see [1]), in insurance models, agents have mainly been assumed to have von-Neumann Morgenstern utilities (denoted v.N.M. from now on). Assuming that the insurer is risk averse and bears a convex cost and that the premium is fixed, efficient contracts are of the following three types: either they entail coinsurance or coinsurance above a deductible or full insurance for low values of the risk and coinsurance after.

However there are insurance indemnity schedules that are commonly offered which don't seem to be efficient for the differentiable von-Neumann Morgenstern model with costs. The contract offered by the FDIC to reinsure American banks againt their losses is a call-spread and gives no reimbursement for low values of the loss, has an upper limit and is linear in between. Swiss health insurance companies provide no reimbursement for small values of the loss and for high values of the loss, the reimbursement equals the loss minus some fixed amount.

This paper argues that the contracts just described are efficient when agents are Rank dependent expected utility maximizers (denoted RDEU from now on). The upper limit or the full insurance above a deductible may be explained by aversion of one of the agent to the worse event. The no reimbursement (full insurance) for low values of the loss follows from the insured (insurer) neglecting events of low probability.

Let us recall that when an agent has a RDEU with a convex distortion $f$ and a utility index $U$, her utility has the following form

$$
E_{f}(U(X))=\left(1-f\left(1_{-}\right)\right) \operatorname{essinf} U(X)+\int_{0}^{1} f^{\prime}(1-t) U\left(F_{X}^{-1}(t)\right) d t
$$

where $f\left(1_{-}\right)=\lim _{x \uparrow 1} f(x)$ and $F_{X}^{-1}$ denotes the quantile of the random variable $X$. The first term takes into account a possible jump at one of $f$ while in the second, the quantile of $X$ is weighted by $f^{\prime}(1-\mathrm{Id})$. Hence aversion to the worse state may be expressed either by assuming that $f$ has a jump at one or by assuming that $\lim _{x \rightarrow 1} f^{\prime}(x)=\infty$. In both cases, the agent highly distorts the probability of very likely events and is very sensitive to the certainty effect.

*Université Paris Dauphine, CEREMADE, UMR 7534, Place de Lattre de Tassigny, 75775 Paris CEDEX 16, FRANCE, carlier@ceremade.dauphine.fr, dana@ceremade.dauphine.fr. 
In order to understand the effect of the jump of $f$ at one, let us first consider the simple case where, for some $\varepsilon \in[0,1], f(x)=(1-\varepsilon) x, x \in[0,1[, f(1)=1$. The jump of $f$ at one equals $\varepsilon$ and

$$
E_{f}(U(X))=\varepsilon \operatorname{essinf} U(X)+(1-\varepsilon) E(U(X))
$$

(2) is closely related to the $\varepsilon$-contaminated expected utility:

$$
u(X):=\varepsilon \inf U(X)+(1-\varepsilon) E_{P}(U(X))
$$

used for example by Dow and Werlang [7], Eichberger and Kelsey [8] and Epstein and Wang [9]. In (3), the agent has multiple priors with set of countably additive priors

$$
\mathcal{P}_{0}:=\{(1-\varepsilon) P+\varepsilon Q: Q \text { countably additive probability measure }\} .
$$

Being uncertainty averse, she minimizes her expected utility over the set of priors $\mathcal{P}_{0}$ and

$$
u(X)=\inf _{R \in \mathcal{P}_{0}} E_{R}(U(X))
$$

while in (2), the agent has a set of countably additive priors

$$
\mathcal{P}:=\{(1-\varepsilon) P+\varepsilon Q: Q \text { absolutely continuous w.r.t. } P\}
$$

and minimizes her expected utility over the set of priors $\mathcal{P}$ and

$$
u(X)=\inf _{R \in \mathcal{P}} E_{R}(U(X)) .
$$

Assuming that both agents have utilities of type (2), with strictly concave utility index and different epsilons, we show that if the insurer takes more into account extreme losses than the insured, then the optimal contract has an upper limit. Equivalently the insurer insures herself a constant wealth for high values of the loss. If the insured takes more into account maximal losses than the insurer, then the optimal indemnity schedule is full insurance minus a deductible for high values of the loss and the insurer insures himself a constant wealth for high values of the loss.

In order to understand the effect of assuming that an agent has a distortion with infinite slope at one, we next study the exchange of risk between RDEU agents with continuous distortions. We show that if the insurer has a distortion with infinite slope at one, while the insured's distortion has finite slope at one, then she offers a contract with an upper limit. If the insured's distortion has infinite slope at one, while the insurer's distortion has finite slope at one, then the insured insures himself a constant wealth for high value of the loss by buying a contract that gives him full insurance minus a deductible, for high value of the loss. The above analysis of course applies to the exchange of risk between a RDEU agent and an E.U. maximizer.

The common feature between the two cases is that the insurer's aversion to the worse event leads to an upper limit on coverage while the insured's aversion to the worse event leads to a deductible for high values of the loss. Let us recall that this type of contracts cannot be obtained if agents are expected utility maximizers with differentiable utilities, since, efficient contracts are strictly increasing whenever state by state constraints are not binding. Upper limits on coverage have been explained by transaction costs, economies of scale in administrative expenses, limited liability of policy holders, informational asymetries between the insurer and the insured (see for example Hubermann et al. [10], Winter [13] and the references included in those papers). Hence our paper provides a new explanation for upper limits.

We also show that if the insured's distortion has slope zero at zero which means that the insured neglects events of low probability and large outcomes, then an optimal contract contains a deductible for low values of the damage. If the insurer neglects events of low probability, then an optimal contract 
provides full insurance for low values of the damage. In an expected utility framework, deductibles on contracts are explained by a loading factor or by costs (see Arrow [1] or Raviv [11]). Hence, we provide here another explanation for deductibles. Therefore, a model with RDEU agents may explain coexistence of a deductible for low values of the risk and an upper limit on the indemnity schedule, according to the relative slopes at 0 or 1 of the distortions of the insurer and the insured (see Safra and Zilcha [12] for an early similar view).

The paper is organised as follows. In section 2, we recall the concept of Choquet integral with respect to a convex distortion of a probability and its properties. In section 3, we introduce an insurance model, recall existence and properties of Pareto optimal contracts when agents have SSD preserving (see definition 1) utilities. We then get to the central part of the paper: section 4 is devoted to the case where agents have epsilon-contaminated utilities and in section 5, we consider the case of Rank dependent expected utility maximizers with continuous distortions. Proofs are gathered in the appendix.

\section{Choquet integral with respect to a convex distortion}

We recall that a capacity on a measurable space $(\Omega, \mathcal{B})$ is a set function $\nu: \mathcal{B} \rightarrow[0,1]$ such that $\nu(\emptyset)=0, \nu(\Omega)=1$ and for all $A, B \in \mathcal{B}, A \subset B$ implies $\nu(A) \leq \nu(B)$. A capacity $\nu$ is convex if for all $A, B \in \mathcal{B}, \nu(A \cup B)+\nu(A \cap B) \geq \nu(A)+\nu(B)$.

Let $(\Omega, \mathcal{B}, P)$ be a probability space. Let $X \in L^{\infty}$ be a random variable and $F_{X}(t)=P(X \leq$ $t), t \in \mathbb{R}$ denote its distribution function. The generalized inverse of $F_{X}$ is defined by:

$$
\left.\left.F_{X}^{-1}(0)=\operatorname{essinf} X \text { and } F_{X}^{-1}(t)=\inf \left\{z \in \mathbb{R}: F_{X}(z) \geq t\right\}, \text { for all } t \in\right] 0,1\right]
$$

A distortion is an increasing map $f:[0,1] \rightarrow[0,1]$ such that $f(0)=0, f(1)=1$. It may easily be verified that $\nu:=f(P)$ is a capacity. Let $X \in L^{\infty}$. The Choquet integral of $X$ with respect to the capacity $f(P)$, denoted $E_{f}(X)$ is defined by

$$
E_{f}(X)=\int_{-\infty}^{0}(f(P(\{X>t\}))-1) d t+\int_{0}^{\infty} f(P(\{X>t\})) d t .
$$

Following Yaari's model, we will also refer to $E_{f}(X)$ as Yaari utility. When $f$ is convex, $\nu=f(P)$ is a convex capacity. Furthermore $f$ is continuous on $\left[0,1\left[\right.\right.$ and differentiable a.e. with $f^{\prime} \in L_{+}^{1}[0,1]$. When $f$ is continuous on $[0,1]$, since $f$ is absolutely continuous, one has:

$$
E_{f}(X)=\int_{0}^{1} f^{\prime}(1-t) F_{X}^{-1}(t) d t
$$

When $f$ is discontinuous, denoting $f\left(1_{-}\right)=\lim _{x \uparrow 1} f(x)$ and $\tilde{f}:=\frac{f}{f\left(1_{-}\right)}$if $x<1$ with $\tilde{f}(1)=1$ (so that $\tilde{f}$ is continuous at 1 ), we then get:

$$
\begin{aligned}
E_{f}(X) & =\int_{0}^{\operatorname{essinf}(X)} f(P(X>t)) d t+\int_{\operatorname{essinf}(X)}^{\infty} f(P(X>t)) d t \\
& =\operatorname{essinf}(X)+f\left(1_{-}\right)\left[\int_{0}^{\infty} \tilde{f}(P(X>t)) d t-\int_{0}^{\operatorname{essinf}(X)} \tilde{f}(P(X>t)) d t\right] . \\
& =\left(1-f\left(1_{-}\right)\right) \operatorname{essinf} X+f\left(1_{-}\right) E_{\tilde{f}}(X) \\
& =\left(1-f\left(1_{-}\right)\right) F_{X}^{-1}(0)+\int_{0}^{1} f^{\prime}(t) F_{X}^{-1}(1-t) d t
\end{aligned}
$$

Let $U: \mathbb{R} \rightarrow \mathbb{R}$ be strictly increasing. The rank dependent expected utility (RDEU) is defined by $v(X)=E_{f}(U(X))$. When $f$ is convex, we have

$$
E_{f}(U(X))=\left(1-f\left(1_{-}\right)\right) U\left(F_{X}^{-1}(0)\right)+\int_{0}^{1} f^{\prime}(1-t) U\left(F_{X}^{-1}(t)\right) d t
$$

Given $(\Omega, \mathcal{B}, P)$, a probability space, let us recall the definition of second order stochastic dominance: 
Definition 1 The random variable $X$ (strictly) dominates $Y$ in the sense of second order stochastic dominance (S.S.D.) denoted $X \succeq_{2} Y$ (resp $X \succ_{2} Y$ ) if any of the following equivalent conditions are fulfilled:

1. $\left.\left.\int_{0}^{t} F_{X}^{-1}(s) d s \geq \int_{0}^{t} F_{Y}^{-1}(s) d s, \forall t \in\right] 0,1\right]$, (resp with a strict inequality for some $t$ ),

2. $E[u(X)] \geq E[u(Y)], \forall u: \mathbb{R} \rightarrow \mathbb{R}$ concave increasing (resp with a strict inequality for some u concave increasing),

3. $Y \stackrel{d}{\sim} X+\varepsilon$ for some $\varepsilon$ such that $E[\varepsilon \mid X] \leq 0$ a.s. (resp where in addition $P(\varepsilon \neq 0)>0$ )

$X$ and $Y$ are S.S.D. equivalent, denoted by $X \sim_{2} Y$, if 1 or 2 holds with equality throughout. In other words, $X \sim_{2} Y$ iff $X \stackrel{d}{\sim} Y$. It follows from condition 3 and Jensen's inequality that $X \succ_{2} Y$ if and only if $E[u(X)]>E[u(Y)]$ for every increasing and strictly concave $u: \mathbb{R} \rightarrow \mathbb{R}$.

Let us then define the notions of monotone and S.S.D. preserving maps:

Definition 2 1. A map $v: L^{\infty}(\Omega) \rightarrow \mathbb{R}$ is (strictly) monotone if $X \geq Y$ a.e. implies $v(X) \geq v(Y)$ (resp $v(X)>v(Y)$ whenever $X \geq Y$ a.e. and $P(X \neq Y)>0$.

2. A map $v: L^{\infty}(\Omega) \rightarrow \mathbb{R} \cup\{-\infty\}$ (strictly) preserves S.S.D. if $X \succeq_{2} Y$ implies $v(X) \geq v(Y)$ (resp $v(X)>v(Y)$ whenever $\left.X \succ_{2} Y\right)$.

S.S.D. preserving maps are not concave in general. Examples of S.S.D. preserving maps which are not concave may be found in Chew et al [3] and Dana [5].

Let us then state some properties of Choquet integrals with respect to a convex distortion. The following proposition is crucial for obtaining existence results. In the case of a continuous distortion, we refer to [2] and [5] for a proof. The case of a distortion with a jump at 1 can easily be derived from an approximation argument.

Proposition 1 Let $X \in L^{\infty}(\Omega)$ and $f$ be a convex distortion. Then

1. $E_{f}$ is concave, monotone $\sigma\left(L^{\infty}, L^{1}\right)$ upper-semi-continuous and S.S.D. preserving. It is strictly S.S.D. preserving if $f$ is strictly convex.

2. If $U: \mathbb{R} \rightarrow \mathbb{R}$ is concave, $v(X)=E_{f}(U(X))$ is concave, monotone $\sigma\left(L^{\infty}, L^{1}\right)$ upper-semicontinuous and S.S.D. preserving. It is strictly S.S.D. preserving if $U$ is strictly concave or $f$ strictly convex.

Let us see now how aversion to the worse state can naturally be modelled in the Yaari framework. Since, for a general convex distortion (continuous or not), we have:

$$
E_{f}(X)=\left(1-f\left(1_{-}\right)\right) \operatorname{essinf} X+\int_{0}^{1} f^{\prime}(1-t) F_{X}^{-1}(t) d t
$$

we can see that the Yaari utility provides two ways of expressing aversion to the worse state: either $\lim _{x \rightarrow 1} f^{\prime}(x)=\infty$ or $f$ has a jump at one. In both cases, the agent with utility $E_{f}$ is very sensitive to the certainty effect since she distorts very much the probability of very likely events. In order to study this effect, we shall pay special attention to the case:

$$
f(x)=(1-\varepsilon) x, x \in[0,1[, f(1)=1, \varepsilon \in[0,1]
$$


where the jump of $f$ at one equals $\varepsilon$, hence we have:

$$
E_{f}(X)=(1-\varepsilon) E_{P}(X)+\varepsilon \operatorname{essinf} X .
$$

The uncertainty aversion of the agent is then quantified by $\varepsilon$.

We shall also study the case where $f$ is continuous with infinite slope of $f$ at one. We shall further discuss the case where agents neglect the highest outcomes (i.e. minimal losses) although we are aware that this assumption is in contradiction with the assumptions of prospect theory or some experiments in behavioral finance.

\section{An insurance Model}

Taken as primitive is a probability space $(\Omega, \mathcal{B}, P)$. A risk averse agent faces a risk $W \in L_{+}^{\infty}(P)$ with values in $[0, b]$, to his initial wealth $w_{0}$. The market provides insurance contracts for this risk. A contract is characterized by a premium $\Pi$ and an indemnity schedule $\bar{I}: \Omega \rightarrow \mathbb{R}_{+}$. A contract is feasible if $0 \leq \bar{I} \leq W P$-a.e.. When the insured buys the contract, she is endowed with the random wealth $X_{2}=w_{0}-\Pi-W+\bar{I}$, while by selling the contract, the insurer gets $\Pi$ and promises to pay $\bar{I}(s)$ if state $s$ occurs. Assuming that her initial wealth is zero, we denote by $X_{1}=\Pi-I$ her wealth. A pair of wealths $\left(X_{1}, X_{2}\right)$ is feasible if $X_{1}+X_{2}=w_{0}-W$ and furthermore $\Pi-W \leq X_{1} \leq \Pi$. We assume that agents' preferences are modeled by monotone utility functions: $u_{i}: L^{\infty}(P) \rightarrow \mathbb{R}$ that we shall further specify. For the sake of completeness, we recall that a feasible pair of wealths $\left(X_{1}, X_{2}\right)$ is Pareto-optimal if there is no other feasible pair $\left(Y_{1}, Y_{2}\right)$ such that $u_{i}\left(Y_{i}\right) \geq u_{i}\left(X_{i}\right)$ for $i=1,2$ with at least one strict inequality.

Let us recall the notion of comonotone pairs of random variables:

Definition $3 A$ pair $(X, Y) \in\left(L^{\infty}\right)^{2}$ is comonotone if there exists a subset $B \subset \Omega \times \Omega$ of measure one for $P \otimes P$ such that

$$
\left[X(s)-X\left(s^{\prime}\right)\right]\left[Y(s)-Y\left(s^{\prime}\right)\right] \geq 0, \forall\left(s, s^{\prime}\right) \in B \times B
$$

Let $W:=X+Y, a:=\operatorname{essinf} W$ and $b:=\operatorname{esssup} W$. An alternative characterization of comonotonicity of a pair of random variables is given by Denneberg [6] :

Lemma 1 A pair $(X, Y) \in\left(L^{\infty}\right)^{2}$ is comonotone iff there exists a pair of non decreasing continuous function $\left(h_{1}, h_{2}\right), h_{i}:[a, b] \rightarrow \mathbb{R}$ such that $h_{1}+h_{2}=I d$ and $X=h_{1}(W)$, a.e. and $Y=h_{2}(W)$ a.e.

We first provide sufficient conditions for existence of Pareto optimal contracts without assuming concavity of utilities. We then show that Pareto optimal contract have properties which are standard when agents are expected utility maximizers: optimal indemnity schedules are non decreasing 1Lipschitz functions of the loss.

Let us introduce the following assumptions:

$\mathbf{U} 1 u_{i}$ are $\sigma\left(L^{\infty}, L^{1}\right)$ upper semi-continuous, $i=1,2$,

U2 $u_{i}, i=1,2$ are S.S.D. preserving and some agent is strictly S.S.D. preserving.

Theorem 1 Assume that utilities fulfill $\mathbf{U 1}$, then

1. there exists Pareto-optimal contracts,

2. if furthermore utilities fulfill $\mathbf{U 2}$, any optimal contract $\bar{I}^{*}$ is such that $\bar{I}^{*}$ and $W-\bar{I}^{*}$ are non decreasing continuous functions of $W$. 
The proof may be found in Carlier and Dana [2]. Let us remark that from proposition 1, theorem 1 applies to both cases of a continuous or discontinuous distortion.

Corollary 1 Assume that agents have Yaari utilities with $f$ convex or RDEU utilities with $f$ convex and $U$ concave. Then there exists optimal contracts. If some agent has a utility with $f$ strictly convex or $U$ strictly concave, then any optimal indemnity schedule $\bar{I}^{*}$ is such that $\bar{I}^{*}$ and $W-\bar{I}^{*}$ are non decreasing continuous functions of $W$.

Theorem 1 implies that Pareto efficient allocations only depend on $W$. We shall from now on use capital letters to denote random variables on $\Omega$ and small letters to denote functions of $W$. Pareto optimal pairs of wealths $\left(X_{1}^{*}, X_{2}^{*}\right)$ can be written as $\left(x_{1}^{*}(W), x_{2}^{*}(W)\right)$ with $x_{i}^{*}:[0, b] \rightarrow \mathbb{R}, i=1,2$ nondecreasing continuous functions, solutions to the following problems:

$$
\left\{\begin{array}{l}
\max v_{1}\left(x_{1}\right) \\
v_{2}\left(x_{2}\right) \geq u_{2}^{*} \\
\Pi-t \leq x_{1}(t) \leq \Pi, \quad \text { for all } t \in[0, b] \\
x_{1}(t)+x_{2}(t)=w_{0}-t, \quad \text { for all } t \in[0, b]
\end{array}\right.
$$

where $v_{i}\left(x_{i}\right):=u_{i}\left(x_{i}(W)\right)$ and $u_{2}^{*}:=u_{2}\left(x_{2}^{*}(W)\right)$.

In the following sections, we shall further specify utilities and describe the qualitative properties of optimal solutions.

\section{Epsilon-contaminated capacities}

We first assume that agents' preferences are represented by the utility functions:

$$
\left\{\begin{array}{l}
u_{1}\left(X_{1}\right)=\left[\left(1-\varepsilon_{1}\right) E_{P}\left[U_{1}\left(X_{1}\right)\right]+\varepsilon_{1} \operatorname{essinf} U_{1}\left(X_{1}\right)\right] \\
u_{2}\left(X_{2}\right)=\left[\left(1-\varepsilon_{2}\right) E_{P}\left[U_{2}\left(X_{2}\right)\right]+\varepsilon_{2} \operatorname{essinf} U_{2}\left(X_{2}\right)\right]
\end{array}\right.
$$

where $U_{i}: \mathbb{R} \rightarrow \mathbb{R}, i=1,2$ is strictly increasing, concave $C^{1}$, strictly concave for some $i$ and $\varepsilon_{i} \in[0,1[, i=1,2$. In other words, the value of a random wealth is a weighted average of its expected utility with respect to the prior $P$ and the utility of the worst possible outcome in probability. In the case, $\varepsilon_{i}=0$, agent $i$ is an expected utility maximizer.

Since efficient contracts are non decreasing and 1-Lipschitz continuous functions of the loss and since essinf $I^{*}(W)=I^{*}(\operatorname{essinf} W)=\min _{[0, b]} I^{*}(t)=I^{*}(b)$, we are therefore reduced to characterize the solutions to the following problem

$$
\left\{\begin{array}{l}
\max \left(1-\varepsilon_{1}\right) E_{Q}\left[U_{1}(\Pi-I(t))\right]+\varepsilon_{1} \min _{t \in[0, b]} U_{1}(\Pi-I(t)) \\
\text { subject to : } \\
\left(1-\varepsilon_{2}\right) E_{Q}\left[U_{2}\left(w_{0}-\Pi-t+I(t)\right)\right]+\varepsilon_{2} \min _{t \in[0, b]} U_{2}\left(w_{0}-\Pi-t+I(t)\right) \geq u_{2}^{*}, \\
0 \leq I(t) \leq t \quad \text { for all } t \in[0, b]
\end{array}\right.
$$

with $I:[0, b] \rightarrow \mathbb{R}^{+}$continuous non decreasing and $Q$ being the probability measure $d F_{W}$ on $([0, b], \mathcal{B})$ and $u_{2}^{*}=\left(1-\varepsilon_{2}\right) E_{Q}\left[U_{2}\left(w_{0}-\Pi-t+I^{*}(t)\right)\right]+\varepsilon_{2} \min _{t \in[0, b]} U_{2}\left(w_{0}-\Pi-t+I^{*}(t)\right)$. Hence agents behave as if they had for set of priors the epsilon contamination of the probability measure on the non-negative reals $d F_{W}$.

We may now characterize optimal contracts. We show that if the insured takes more into account maximal losses than the insurer, then the optimal indemnity schedule equals the loss minus a deductible for high values of the loss, in other words the insured insures herself a constant wealth. On the contrary, if the insurer takes more into account maximal losses than the insured, then the optimal contract has an upper limit. This phenomenon holds true independently of agents' utility index. Agents' utility index however determine the policy for low values of the loss and the level of the upper limit.

In the sequel, we shall denote by $\delta_{x}$ the Dirac measure at point $x$. 
Theorem 2 Assume that $d F_{W}=(1-p) f_{W}(x) d x+p \delta_{0}$, with $p \in\left[0,1\left[\right.\right.$ and that $U_{i}, i=1,2$ is strictly concave, then

- Assume that $\varepsilon_{2}>\varepsilon_{1}$. If a contract I is Pareto-optimal, then

1. either there exists $D \leq b, k \leq b$ such that $I(t)=t-D, \forall t \in[k, b]$. In other words, an optimal contract includes a deductible for high values of the loss. On $[0, k], I$ is the restriction of an efficient contract of Expected utility maximizers with same probability and utility index $\left(1-\varepsilon_{i}\right) U_{i}, i=1,2$.

2. or $I(t)=0$ for all $t \in[0, b]$.

- Assume $\varepsilon_{1}>\varepsilon_{2}$. If a contract I is Pareto-optimal, then

1. either there exists $D$ and $k$ with $D \leq k \leq b$ such that $I(t)=D, \forall t \in[k, b]$. In other words an optimal contract has an upper limit. On $[0, k], I$ is the restriction of an efficient contract of Expected utility maximizers with same probability and utility index $\left(1-\varepsilon_{i}\right) U_{i}, i=1,2$.

2. or $I(t)=t$ for all $t \in[0, b]$.

The proof may be found in the appendix. In the case where one agent has a linear index, a more precise statement may be made.

Proposition 2 Let the insurer have utility $u_{1}\left(X_{1}\right)=\left[\left(1-\varepsilon_{1}\right) E_{P}\left(X_{1}\right)+\varepsilon_{1}\right.$ essinf $\left.X_{1}\right]$ and the insured have utility $u_{2}\left(X_{2}\right)=\left[\left(1-\varepsilon_{2}\right) E_{P} U_{2}\left(X_{2}\right)+\varepsilon_{2}\right.$ essinf $\left.U_{2}\left(X_{2}\right)\right]$ with $\varepsilon_{2} \geq \varepsilon_{1}$ where $U_{2}: \mathbb{R} \rightarrow \mathbb{R}$ is strictly concave, strictly increasing and $C^{1}$. If a contract $I:[0, b] \rightarrow \mathbb{R}$ is Pareto-optimal, then there exists $D$ such that $I=(I d-D)^{+}$. If $u_{1}\left(X_{1}\right)=\left[\left(1-\varepsilon_{1}\right) E_{P}\left(U_{1}\left(X_{1}\right)\right)+\varepsilon_{1}\right.$ essinf $\left.U\left(X_{1}\right)\right]$ and $u_{2}\left(X_{2}\right)=\left[\left(1-\varepsilon_{2}\right) E_{P}\left(X_{2}\right)+\varepsilon_{2}\right.$ essinf $\left.X_{2}\right]$ with $\varepsilon_{1}>\varepsilon_{2}$ where $U_{1}: \mathbb{R} \rightarrow \mathbb{R}$ is strictly concave, strictly increasing and $C^{1}$, then agents do not trade.

The proof may be found in the appendix.

\section{The case of continuous distortions}

The insurance market is as in section two but we now assume that agents' preferences are modelled by RDEU utility functions:

$$
\left\{\begin{array}{l}
u_{1}\left(X_{1}\right)=E_{f_{1}}\left(U_{1}\left(X_{1}\right)\right) \\
u_{2}\left(X_{2}\right)=E_{f_{2}}\left(U_{2}\left(X_{2}\right)\right)
\end{array}\right.
$$

where $U_{i}: \mathbb{R} \rightarrow \mathbb{R}_{+}, i=1,2$ are $C^{1}$, and strictly concave increasing, $f_{i}:[0,1] \rightarrow[0,1], i=1,2$ satisfy $f_{i}(0)=0, f_{i}(1)=1$ and $f_{i}$ are increasing, convex and differentiable on $[0,1[$. In this section, we assume that $(\Omega, \mathcal{B}, P)$ is non-atomic.

Definition 4 Let $(\Omega, \mathcal{B}, P)$ be a probability space. A measurable set $A \in \mathcal{B}$ is an atom of $P$ if $P(A)>0$ and $B \subset A$ implies $P(B)=0$ or $P(A)=P(B)$. A probability space $(\Omega, \mathcal{B}, P)$ is non-atomic if it has no atoms.

In order to characterize optimal wealths, let us first recall a few definitions.

Definition 5 A function $g: L^{\infty} \rightarrow \mathbb{R}$ is Gateaux-differentiable at $X$ if for all $Y \in L^{\infty}$, the limit

$$
D g(X)(Y)=\lim _{t \rightarrow 0^{+}} \frac{1}{t}(g(X+t Y)-g(X))
$$

exists and $Y \mapsto D g(X)(Y)$ is a continuous linear form in the norm topology. 
As $E_{f}$ is concave, we recall that the superdifferential $\partial E_{f}(X)$ of $E_{f}$ at $X \in L^{\infty}$ is defined by

$$
\partial E_{f}(X):=\left\{h \in L^{1} \text { s. t. } E_{f}(X)-E_{f}\left(X^{\prime}\right) \geq \int_{\Omega} h\left(X-X^{\prime}\right) d P, \text { for all } X^{\prime} \in L^{\infty}\right\}
$$

We refer to Carlier and Dana [2] for details and in particular the reason why the superdifferential is included in $L^{1}$ when $f$ is continuous.

Proposition 3 Let $X \in L^{\infty}$, then

1. $E_{f}(U)$ is Gateaux-differentiable at $X$ if and only if $F_{X}$ is continuous and we have:

$$
D E_{f}(U(X))(Y)=\int_{\Omega} f^{\prime}\left(1-F_{X}(X)\right) U^{\prime}(X) Y d P, \forall Y \in L^{\infty}
$$

2. More generally, the superdifferential of $E_{f}(U(X))$ is the closed convex hull for the $L^{1}$ topology of all $f^{\prime}(1-Z) U^{\prime}(X)$ with $Z$ uniformly distributed and such that $Y=F_{Y}^{-1} \circ Z$.

3. Elements of the superdifferential of $E_{f}(U(X))$ coincide on the set of $\omega$ 's such that $P(X=$ $X(\omega)) \neq 0$ and are equal to $f^{\prime}\left(1-F_{X}(X(\omega))\right)$.

The proof of those statements may be found in Carlier and Dana [2]. We may now characterize efficient contracts. We shall consider the case of two RDEU agents, the case of a RDEU agent who exchanges with an expected utility maximizer and finally the case of a RDEU agent who exchanges with an agent with an epsilon-contaminated utility. What plays an important role in the discussion that follows is whether one of the agent has a distortion with infinite slope at one.

The next two results show that if the insurer is very sensitive to the certainty effect (in other words if $\left.f_{1}^{\prime}(1)=\infty\right)$ while the insured is not so $\left(f_{2}^{\prime}(1)<\infty\right)$, then she insures herself a minimal wealth by offering a contract with an upper limit. On the contrary, if the insured is very sensitive to the certainty effect $f_{2}^{\prime}(1)=\infty$ while the insurer is less sensitive $f_{1}^{\prime}(1)<\infty$, then she insures himself a constant wealth for high value of the loss by buying a contract with a deductible. The phenomenon holds true independently of agents' utility index. Agents' utility index determine the policy for low values of the loss and the level of the upper limit.

Proposition 4 Let $f_{i}, i=1,2$ be strictly convex and $U_{i}, i=1,2$ be strictly concave and $F_{W}$ be continuous.

- Assume that $f_{1}^{\prime}(1)=\infty$ and $f_{2}^{\prime}(1)<\infty$. If a contract $I$ is Pareto-optimal, then there exists $D, k \leq b$ such that $I(t)=D, \forall t \in[k, b]$. In other words an optimal contract is constant for high values of the loss.

- Assume that $f_{2}^{\prime}(1)=\infty$ and $f_{1}^{\prime}(1)<\infty$. If a contract $I(t)$ is Pareto-optimal, then there exists $D \leq a, k \leq b$ such that $I(t)=t-D, \forall t \in[k, b]$. In other words an optimal contract includes a deductible for high values of the loss.

The proof may be found in the appendix.

The result stated above also holds true in the case one agent is an expected utility maximizer:

Proposition 5 Let $U_{i}, i=1,2$ be strictly concave and $F_{W}$ be continuous.

- Let $f_{1}$ be strictly convex fulfilling $f_{1}^{\prime}(1)=\infty$ and let $f_{2}(x)=x$. If a contract $I$ is Pareto-optimal, then there exists $D, k \leq b$ such that $I(t)=D, \forall t \in[k, b]$. In other words an optimal contract has an upper limit. 
- Let $f_{2}$ be strictly convex fulfilling $f_{2}^{\prime}(1)=\infty$ and let $f_{1}(x)=x$. If a contract $I$ is Pareto-optimal, then there exists $D, k \leq b$ such that $I(t)=t-D, \forall t \in[k, b]$. In other words an optimal contract includes a deductible for high values of the loss.

Let us next prove that this model allows for coexistence of a deductible for low values of the loss and an upper limit for high values of the loss. The relative slopes of the distortions at zero play an important role in the proof.

Proposition 6 Let $f_{i}, i=1,2$ be strictly convex and $U_{i}, i=1,2$ be strictly concave and $F_{W}$ be continuous.

- Assume that $f_{1}^{\prime}(0)>0$ and $f_{2}^{\prime}(0)=0$. If a contract $I$ is Pareto-optimal, then $I(t)=0$ for $t$ small enough.

- Assume that $f_{2}^{\prime}(0)>0, f_{1}^{\prime}(0)=0$. If a contract $I$ is Pareto-optimal, then $I(t)=t$ for $t$ small enough.

We finally study the case where one agent is RDEU and the other has an epsilon contaminated utility. We only study the case of a RDEU insurer who exchanges risk with an insured with epsilon contaminated utility. The symmetrical case follows from the same logic. Agents' preferences are represented by the utility functions:

$\left\{\begin{array}{l}u_{1}\left(X_{1}\right)=E_{f_{1}}\left[U_{1}\left(X_{1}\right)\right] \\ u_{2}\left(X_{2}\right)=\left[(1-\varepsilon) E_{P}\left[U_{2}\left(X_{2}\right)\right]+\varepsilon \operatorname{essinf} U_{2}\left(X_{2}\right)\right]\end{array}\right.$

where $U_{i}: \mathbb{R} \rightarrow \mathbb{R}, i=1,2$ is strictly increasing, concave $C^{1}$, strictly concave, $f_{i}$ is strictly convex increasing and $C^{1}$ and $\left.\varepsilon \in\right] 0,1[$.

We show that if the sensitivity to the certainty effect of the first agent is moderate, then we get the same qualitative properties as in Proposition 4. If it is high, than the insurer may refuse to sell a contract. If not, she offers a contract with a deductible for high values of the risk.

Proposition 7 Assume $F_{W}$ continuous.

- Assume that $f_{1}^{\prime}(1)<\infty$. If a contract $I^{*}$ is Pareto-optimal, then there exists $0 \leq D \leq b, 0 \leq k \leq$ $b$ such that $I^{*}(t)=t-D, \forall t \in[k, b]$. In other words an optimal contract includes a deductible for high values of the loss.

- Assume that $f_{1}^{\prime}(1)=\infty$. If a contract $I^{*}(t)$ is Pareto-optimal, then, either $I^{*}(t)=0$ or there exists $0 \leq D \leq b, 0 \leq k \leq b$ such that $I^{*}(t)=t-D, \forall t \in[k, b]$.

The proof may be found in the appendix.

\section{Appendix: Proofs}

\section{Proof of Theorem 2}

Assume that $\varepsilon_{2}>\varepsilon_{1}$ and let $I^{*}:[0, b] \rightarrow \mathbb{R}$ be a Pareto optimal contract and let $x_{i}^{*}:[0, b] \rightarrow \mathbb{R}, i=1,2$ be the associated wealths, $x_{1}^{*}(t)=\Pi-I^{*}(t)$ and $x_{2}^{*}(t)=w_{0}-\Pi-t+I^{*}(t)$. There exists $\lambda \geq 0$ such that $I^{*}$ maximizes the functional $F_{\lambda}$ defined below subject to the constraint $0 \leq I(t) \leq t$

$$
\begin{gathered}
F_{\lambda}(I)=\left(1-\varepsilon_{1}\right) E_{Q}\left(U_{1}(\Pi-I)\right)+\varepsilon_{1} \min _{t \in[0, b]} U_{1}(\Pi-I) \\
+\lambda\left[\left(1-\varepsilon_{2}\right) E_{Q}\left(U_{2}\left(w_{0}-\Pi-\mathrm{Id}+I\right)\right)+\varepsilon_{2} \min _{t \in[0, b]} U_{2}\left(w_{0}-\Pi-\mathrm{Id}+I\right)\right]
\end{gathered}
$$


If $\lambda=0$ then $I=0$ and there is nothing to prove we therefore assume $\lambda>0$. First order condition can be written as:

$$
\left(1-\varepsilon_{1}\right) U_{1}^{\prime}\left(x_{1}^{*}\right) d F_{W}+\varepsilon_{1} U_{1}^{\prime}\left(x_{1}^{*}(b)\right) m_{1}-\nu_{1}=\lambda\left[\left(1-\varepsilon_{2}\right) U_{2}^{\prime}\left(x_{2}^{*}\right) d F_{W}+\varepsilon_{2} U_{2}^{\prime}\left(x_{2}^{*}(b)\right) m_{2}\right]-\nu_{2}
$$

where $m_{i}$ is a probability measure for $i=1,2$ that is supported by $\left\{t \in[0, b]\right.$ s.t. $\left.x_{i}^{*}(t)=x_{i}^{*}(b)\right\}$ and $\nu_{i}$ is a nonnegative measure such that $\nu_{1}$ (respectively $\nu_{2}$ ) is supported by $\left\{I^{*}=0\right\}$ (respectively $\left.\left\{I^{*}(t)=t\right\}\right)$.

If both $x_{1}^{*}$ and $x_{2}^{*}$ achieve their minimum only at $b$, then $m_{1}=m_{2}=\delta_{b}$. Furthermore, this implies that $0<I^{*}(b)<b$ and since $I^{*}$ is continuous that $0<I^{*}(t)<t$ for $t$ close to $b$. Hence the measures $\nu_{i}$ vanish in a neighbourhood of $b$. In such a neighbourhood, it follows from (4) that:

$$
\left(1-\varepsilon_{1}\right) U_{1}^{\prime}\left(x_{1}^{*}(t)\right) d F_{W}(t)=\lambda\left[\left(1-\varepsilon_{2}\right) U_{2}^{\prime}\left(x_{2}^{*}(t)\right) d F_{W}(t)\right]
$$

hence

$$
\left(1-\varepsilon_{1}\right) U_{1}^{\prime}\left(x_{1}^{*}(t)\right)=\lambda\left(1-\varepsilon_{2}\right) U_{2}^{\prime}\left(x_{2}^{*}(t)\right)
$$

By continuity, we obtain

$$
\left(1-\varepsilon_{1}\right) U_{1}^{\prime}\left(x_{1}^{*}(b)\right)=\lambda\left(1-\varepsilon_{2}\right) U_{2}^{\prime}\left(x_{2}^{*}(b)\right)
$$

Furthermore, it also follows from (4) that

$$
\varepsilon_{1} U_{1}^{\prime}\left(x_{1}^{*}(b)\right)=\lambda \varepsilon_{2} U_{2}^{\prime}\left(x_{2}^{*}(b)\right)
$$

Dividing (7) by (8), we obtain $\frac{\varepsilon_{1}}{1-\varepsilon_{1}}=\frac{\varepsilon_{2}}{1-\varepsilon_{2}}$, hence $\varepsilon_{1}=\varepsilon_{2}$ a contradiction. Hence $x_{1}^{*}$ and $x_{2}^{*}$ cannot both achieve their minimum only at $b$. Hence one of those functions has to be constant in a neighborhood of $b$.

Assume that $x_{1}^{*}$ is constant in a neighborhood of $b$. We denote by $[k, b]$ the largest interval on which $x_{1}^{*}=x_{1}^{*}(b)$. There are two cases : either $I^{*}(b)=0$ so that $I^{*}(t)=0$ for all $t$ or $0<I^{*}(b)=I^{*}(k) \leq k$. In that case we have $m_{2}=\delta_{b}, \nu_{1}([k, b])=0$ and $\left.\left.\nu_{2}(] k, b\right]\right)=0$. Then using (4), we first get:

$$
\varepsilon_{1} U_{1}^{\prime}\left(x_{1}^{*}(b)\right) \geq \varepsilon_{1} U_{1}^{\prime}\left(x_{1}^{*}(b)\right) m_{1}(\{b\})=\lambda \varepsilon_{2} U_{2}^{\prime}\left(x_{2}^{*}(b)\right)
$$

Similarly we have

$$
0 \geq-\nu_{2}(\{k\})=\varepsilon_{1} U_{1}^{\prime}\left(x_{1}^{*}(b)\right) m_{1}(\{k\})
$$

so that $\nu_{2}(\{k\})=m_{1}(\{k\})=0$.

Integrating (4) between $k$ and $b$, we get:

$$
\lambda\left[\left(1-\varepsilon_{2}\right) \int_{k}^{b} U_{2}^{\prime}\left(x_{2}^{*}(s)\right) d F_{W}+\varepsilon_{2} U_{2}^{\prime}\left(x_{2}^{*}\right)(b)\right]=\left[\left(1-F_{W}(k)\right)\left(1-\varepsilon_{1}\right)+\varepsilon_{1}\right] U_{1}^{\prime}\left(x_{1}^{*}(b)\right)
$$

Since $U_{2}^{\prime}$ and $x_{2}^{*}$ are decreasing, and using (9) we get:

$$
\begin{aligned}
\lambda\left(1-\varepsilon_{2}\right) \int_{k}^{b} U_{2}^{\prime}\left(x_{2}^{*}(s)\right) d F_{W} & <\lambda\left(1-F_{W}(k)\right)\left(1-\varepsilon_{2}\right) U_{2}^{\prime}\left(x_{2}^{*}(b)\right) \\
& \leq\left(1-F_{W}(k)\right)\left(1-\varepsilon_{2}\right) \frac{\varepsilon_{1}}{\varepsilon_{2}} U_{1}^{\prime}\left(x_{1}^{*}(b)\right)
\end{aligned}
$$

then using (10) and (9), we obtain:

$$
\begin{aligned}
{\left[\left(1-F_{W}(k)\right)\left(1-\varepsilon_{1}\right)+\varepsilon_{1}\right] U_{1}^{\prime}\left(x_{1}^{*}(b)\right) } & <\left[\frac{\varepsilon_{1}}{\varepsilon_{2}}\left(1-\varepsilon_{2}\right)\left(1-F_{W}(k)\right)+\varepsilon_{1}\right] U_{1}^{\prime}\left(x_{1}^{*}(b)\right) \\
& =\left[\left(1-F_{W}(k)\right) \frac{\varepsilon_{1}}{\varepsilon_{2}}+\varepsilon_{1} F_{W}(k)\right] U_{1}^{\prime}\left(x_{1}^{*}(b)\right)
\end{aligned}
$$

After simple computations, this last inequality yields $\varepsilon_{1}>\varepsilon_{2}$, hence a contradiction and $x_{1}^{*}$ cannot be constant in a neighborhood of $b$. Finally this proves that either $I^{*}$ is the zero function or $x_{2}^{*}$ is constant on some interval $[k, b]$. In that case, it follows from (4) that the restriction of $I^{*}$ to $[0, k[$ is a Pareto optimal contract of expected utility maximizers with utilities $\left(1-\varepsilon_{i}\right) U_{i}$ 


\section{Proof of Proposition 2}

Let $I:[0, b] \rightarrow \mathbb{R}$ be a feasible contract and let $\left(x_{1}, x_{2}\right)$ be the associated wealths. Let us prove that $I$ is dominated by a contract $(\operatorname{Id}-D)^{+}$for some $D$. From Corollary 1 , we can assume that $I(W)$ and $W-I(W)$ are non decreasing functions of $W$. If $I$ is not of the form $(\operatorname{Id}-D)^{+}$for some $D$, let $D$ be such that $v_{1}\left(x_{1}\right)=v_{1}\left(\Pi-(\operatorname{Id}-D)^{+}\right)$. Equivalently $E_{Q^{\prime}}\left(x_{1}\right)=E_{Q^{\prime}}\left(\Pi-(\operatorname{Id}-D)^{+}\right)$with $Q^{\prime}=\left(1-\varepsilon_{1}\right) Q+\varepsilon_{1} \delta_{b}$. Let $x_{2 D}(t)=w_{0}-\Pi-t+(t-D)^{+}=w_{0}-\Pi-\min (t, D)$. As $U_{2}$ is strictly concave,

$$
E_{Q^{\prime}}\left(U_{2}\left(x_{2 D}\right)-E_{Q^{\prime}}\left(U_{2}\left(x_{2}\right)\right)>U_{2}^{\prime}\left(w_{0}-\Pi-D\right)\left[\left(E_{Q^{\prime}}\left(x_{1}\right)-E_{Q^{\prime}}\left(\Pi-(\operatorname{Id}-D)^{+}\right)\right)=0\right.\right.
$$

In other words,

$$
\left(1-\varepsilon_{1}\right) E_{Q}\left(U_{2}\left(\left(x_{2 D}\right)\right)+\varepsilon_{1} U_{2}\left(\left(x_{2 D}(b)\right)>\left(1-\varepsilon_{1}\right) E_{Q}\left(U_{2}\left(x_{2}\right)\right)+\varepsilon_{1} U_{2}\left(x_{2}(b)\right)\right.\right.
$$

Let us show that $D<b-I(b)$ or equivalently that $x_{2 D}(b)>x_{2}(b)$. Indeed $D \geq b-I(b)$ implies $D \geq t-I(t)$ for all $t \in[0, b]$, hence $\min (t, D) \geq t-I(t)$ for all $t \in[0, b]$ or $x_{2 D}(t) \leq x_{2}(t)$ for all $t$ and $E_{Q^{\prime}}\left(U_{2}\left(x_{2 D}\right)\right) \leq E_{Q^{\prime}}\left(U_{2}\left(x_{2}\right)\right)$ contradicting (13). Hence $D<b-I(b)$. Therefore $x_{2 D}(b)=w_{0}-\Pi-D>x_{2}(b)$.

There are two cases: either

$$
E_{Q}\left(U_{2}\left(x_{2 D}\right)\right) \geq E_{Q}\left(U_{2}\left(x_{2}\right)\right) .
$$

then obviously we have $v_{2}\left(x_{2 D}\right)>v_{2}\left(x_{2}\right)$ which proves that $(\mathrm{Id}-D)^{+}$dominates $I$, or:

$$
\begin{aligned}
E_{Q}\left(U_{2}\left(x_{2 D}\right)\right) & <E_{Q}\left(U_{2}\left(x_{2}\right)\right) \\
\text { As } \quad \varepsilon_{2}\left[U_{2}\left(x_{2 D}(b)\right)-U_{2}\left(X_{2}(b)\right)\right] & >\varepsilon_{1}\left[U_{2}\left(x_{2 D}(b)\right)-U_{2}\left(X_{2}(b)\right)\right] \\
& >\left(1-\varepsilon_{1}\right)\left[E_{Q}\left(U_{2}\left(X_{2}\right)\right)-E_{Q}\left(U_{2}\left(x_{2 D}\right)\right)\right] \\
& >\left(1-\varepsilon_{2}\right)\left[E_{Q}\left(U_{2}\left(X_{2}\right)\right)-E_{Q}\left(U_{2}\left(x_{2 D}\right)\right)\right]
\end{aligned}
$$

(the first inequality follows from $x_{2 D}(b)>x_{2}(b)$ and $\varepsilon_{2} \geq \varepsilon_{1}$ and $U_{2}$ increasing, the second from (14)) and the third from (14) and $\varepsilon_{2} \geq \varepsilon_{1}$. We therefore get

$$
\left.\left(1-\varepsilon_{2}\right) E_{Q}\left(U_{2}\left(x_{2 D}\right)\right)+\varepsilon_{2} U_{2}\left(x_{2 D}(b)\right)>\left(1-\varepsilon_{2}\right) E_{Q}\left(U_{2}\left(x_{2}\right)\right)\right)+\varepsilon_{2} U_{2}\left(x_{2}(b)\right)
$$

which proves that $(\mathrm{Id}-D)^{+}$dominates $I$

\section{Proof of Proposition 4}

We prove the result in the case : $f_{1}^{\prime}(1)=+\infty, f_{2}^{\prime}(1)<+\infty$. The other case is obviously obtained by permuting the two agents.

Let $I^{*}:[0, b] \rightarrow \mathbb{R}$ be a Pareto optimal contract and let $x_{i}^{*}:[0, b] \rightarrow \mathbb{R}, i=1,2$ be the associated wealths, $x_{1}^{*}(t)=\Pi-I^{*}(t)$ and $x_{2}^{*}(t)=w_{0}-\Pi-t+I^{*}(t)$. Since $I^{*}$ is Pareto-Optimal, there exists $\lambda \geq 0$ such that $I^{*}$ maximizes

$$
F_{\lambda}(I):=E_{f_{1}}\left(U_{1}(\Pi-I(W))\right)+\lambda E_{f_{2}}\left(U_{2}\left(w_{0}-\Pi+I(W)-W\right)\right)
$$

subject to the constraint $0 \leq I \leq W$.

Let us first prove that there exists a neighbourhood of $b$ on which $I^{*}(t)<t$. If it was not the case, as $t \mapsto I^{*}(t)-t$ is nonincreasing, one would have $I^{*}(t)=t$ for all $t \in[0, b]$. Let us therefore show that $I^{*} \neq \mathrm{Id}$. For $\varepsilon>0$ small enough, let

$$
I_{\varepsilon}(t):=\min (t, b-\varepsilon)
$$


We have

$$
E_{f_{2}}\left(U_{2}\left(w_{0}-\Pi+I_{\varepsilon}(W)-W\right)\right)-E_{f_{2}} U_{2}\left(w_{0}-\Pi\right) \geq \int_{\Omega} h\left(I_{\varepsilon}(W)-W\right) U_{2}^{\prime}\left(w_{0}-\Pi+I_{\varepsilon}(W)-W\right) d P
$$

for any $h \in \operatorname{core}\left(f_{2}(P)\right)$. Since $h \leq f_{2}^{\prime}(1)$ and $I_{\varepsilon}(W)-W \leq 0$, we get

$$
\begin{aligned}
E_{f_{2}}\left(U_{2}\left(w_{0}-\Pi+I_{\varepsilon}(W)-W\right)\right)-E_{f_{2}} U_{2}\left(w_{0}-\Pi\right) & \geq \int_{b-\varepsilon}^{b} f_{2}^{\prime}(1) U_{2}^{\prime}\left(w_{0}-\Pi+b-\varepsilon-t\right)(b-\varepsilon-t) d F_{W}(t) \\
& \geq U_{2}^{\prime}\left(w_{0}-\Pi-\varepsilon\right) f_{2}^{\prime}(1) \int_{b-\varepsilon}^{b}(b-\varepsilon-t) d F_{W}(t)
\end{aligned}
$$

Similarly we have

$$
E_{f_{1}} U_{1}\left(\Pi-I_{\varepsilon}(W)\right)-E_{f_{1}} U_{1}(\Pi-W) \geq \int_{\Omega} h\left(W-I_{\varepsilon}(W)\right) U_{1}^{\prime}\left(\Pi-I_{\varepsilon}\right) d P
$$

for any $h \in \partial E_{f_{1}}\left(U_{1}\left(\Pi-I_{\varepsilon}\right)\right)$. Taking $h=f_{1}^{\prime}\left(F_{W}(W)\right)$ in (19), we obtain

$$
\begin{aligned}
E_{f_{1}}\left(U_{1}\left(\Pi-I_{\varepsilon}(W)\right)\right)-E_{f_{1}} U_{1}(\Pi-W) & \geq \int_{b-\varepsilon}^{b} f_{1}^{\prime}\left(F_{W} t\right) U_{1}^{\prime}(\Pi-b+\varepsilon)(t-b+\varepsilon) d F_{W}(t) \\
& \geq f_{1}^{\prime}\left(F_{W}(b-\varepsilon)\right) U_{1}^{\prime}(\Pi-b+\varepsilon) \int_{b-\varepsilon}^{b}(t-b+\varepsilon) d F_{W}(t)
\end{aligned}
$$

Since $f_{1}^{\prime}\left(F_{W}(t)\right)$ tends to $+\infty$ as $t$ tends to $b$, it follows from (18) and (20) that

$$
F_{\lambda}\left(I_{\varepsilon}\right)-F_{\lambda}(\mathrm{Id}) \geq\left(f_{1}^{\prime}\left(F_{W}(b-\varepsilon)\right) U_{1}^{\prime}(\Pi-b+\varepsilon)-\lambda U_{2}^{\prime}\left(w_{0}-\Pi-\varepsilon\right) f_{2}^{\prime}(1)\right) \int_{b-\varepsilon}^{b}(t-b+\varepsilon) d F_{W}(t)>0
$$

for $\varepsilon>0$ small enough. Hence $I^{*} \neq \mathrm{Id}$.

Let us show now that $x_{1}^{*}$ and $x_{2}^{*}$ cannot be both strictly decreasing. Since $x_{i}^{*}$ strictly decreasing implies that $F_{x_{i}^{*}}^{-1}(t)=x_{i}^{*} \circ F_{W}^{-1}(1-t)$ is strictly increasing, we may therefore apply proposition 3 . As $F_{x_{i}^{*}}\left(x_{i}^{*}\right)=1-F_{W}(W)$, the first order conditions are,

$$
\lambda U_{2}^{\prime}\left(x_{2}^{*}(W(\omega))\right) f_{2}^{\prime}\left(F_{W}\left(x_{2}^{*}(W(\omega))\right)\right)=U_{1}^{\prime}\left(\left(x_{1}^{*}(W(\omega))\right) f_{1}^{\prime}\left(F_{W}(W(\omega))\right), \forall \omega\right.
$$

Equivalently,

$$
\lambda U_{2}^{\prime}\left(x_{2}^{*}(t)\right) f_{2}^{\prime}\left(F_{W}(t)\right)=U_{1}^{\prime}\left(x_{1}^{*}(t)\right) f_{1}^{\prime}\left(F_{W}(t)\right), \forall t \in[0, b]
$$

Let $t \rightarrow b$. Since $U_{i}^{\prime}$ is strictly decreasing and $x_{i}^{*}$ is decreasing, we have $U_{i}^{\prime}\left(x_{i}^{*}(b)\right)>0, i=1,2$. As $f_{1}^{\prime}\left(F_{W}(t)\right) \rightarrow \infty, f_{2}^{\prime}\left(F_{W}(t)\right) \rightarrow \infty$ contradicting the hypothesis. Hence $x_{i}^{*}, i=1,2$ cannot be both strictly decreasing. Let us now show that $x_{1}^{*}$ is constant in a neighbourhood of $b$. By proposition 3 , the first order conditions are:

$$
f_{1}^{\prime}\left(Z_{1}(\omega)\right) U_{1}^{\prime}\left(x_{1}^{*}(W(\omega))\right)=\lambda f_{2}^{\prime}\left(Z_{2}(\omega)\right) U_{2}^{\prime}\left(x_{2}^{*}(W(\omega))\right)
$$

with $Z_{i}(\omega)=F_{W}(W(\omega))$ for all $\omega$ which does not belong to a level set of $x_{i}^{*}(w)$ with positive measure. If $x_{1}^{*}$ is not constant in a neighbourhood of $b$, then there exists an increasing sequence $w_{n}$ with limit $b$ and such that for almost every $\omega \in\left\{W=w_{n}\right\}, Z_{1}(\omega)=F_{W}\left(w_{n}\right)$. Hence for every $n$, we have:

$$
\lambda f_{2}^{\prime}\left(s_{2}(\omega)\right) U_{2}^{\prime}\left(x_{2}^{*}(W(\omega))\right)=U_{1}^{\prime}\left(x_{1}^{*}\left(w_{n}\right)\right) f_{1}^{\prime}\left(F_{W}\left(w_{n}\right)\right), \forall \omega \in\left\{W=w_{n}\right\}
$$

Passing to the limit, we have a contradiction the right hand side being unbounded while the left hand side is bounded. Hence $x_{1}^{*}$ is constant in a neighbourhood of $b$ 


\section{Proof of Proposition 5}

The proof of the first case is as in the previous proposition. One first shows that there exists a neighborhood $[b-\varepsilon, b]$ of $b$ such that $I(t)<t$, for all $t \in[b-\varepsilon, b]$. One then shows that $x_{1}^{*}$ and $x_{2}^{*}$ cannot be both strictly decreasing since the first order conditions would be:

$$
\lambda f_{1}^{\prime}\left(F_{W}(t)\right) U_{1}^{\prime}\left(x_{1}^{*}(t)\right)=U_{2}^{\prime}\left(x_{2}^{*}(t)\right), \forall t \in[0, b]
$$

leading to a contradiction as $t \rightarrow b$ since the left hand side tends to $\infty$ and the right hand side to a finite value. The end of the proof is similar to that of proposition 4

\section{Proof of Proposition 6}

Let $I^{*}:[0, b] \rightarrow \mathbb{R}$ be a Pareto optimal contract. Let us first show that there does not exist $\alpha>0$ such that $I^{*}(t)=t$ on $[0, \alpha]$.

Indeed, assume by contradiction that $I^{*}$ is Pareto-Optimal and $I^{*}(t)=t$ on $[0, \alpha]$ for some $\alpha>0$. Since $I^{*}$ is Pareto-Optimal, there exists $\lambda \geq 0$ such that $I^{*}$ maximizes

$$
F_{\lambda}(I):=E_{f_{1}}\left(U_{1}(\Pi-I(W))\right)+\lambda E_{f_{2}}\left(U_{2}\left(w_{0}-\Pi+I(W)-W\right)\right)
$$

subject to the constraint $0 \leq I \leq W$.

For $\varepsilon \in(0, \alpha)$, let

$$
I_{\varepsilon}(t):=I(t) 1_{t \geq \varepsilon}
$$

Let $x_{1 \varepsilon}=\Pi-I_{\varepsilon}$ and $x_{2 \varepsilon}=w_{0}-\Pi+I_{\varepsilon}-$ Id be the associated wealths. As $x_{2 \varepsilon}=w_{0}-\Pi-$ Id in a neighborhood of 0 , it is strictly decreasing in that neighborhood. By proposition 3 , the function $U_{2}^{\prime}\left(w_{0}-\Pi-W\right) f_{2}^{\prime}\left(F_{W}(W)\right.$ is the only element in the restriction of $\partial E_{f_{2}}\left(U_{2}\left(w_{0}-\Pi+I_{\varepsilon}(W)-W\right)\right.$ to $\{W \leq \varepsilon\}$ (see Carlier and Dana[2]). As $I_{\varepsilon}=I$ for $t \geq \varepsilon$, we thus have:

$E_{f_{2}}\left(U_{2}\left(w_{0}-\Pi+I_{\varepsilon}(W)-W\right)\right)-E_{f_{2}}\left(U_{2}\left(w_{0}-\Pi+I(W)-W\right)\right) \geq-\int_{0}^{\varepsilon} t U_{2}^{\prime}\left(-t+w_{0}-\Pi\right) f_{2}^{\prime}\left(F_{W}(t)\right) d F_{W}(t)$

hence

$E_{f_{2}}\left(U_{2}\left(w_{0}-\Pi+I_{\varepsilon}(W)-W\right)-E_{f_{2}}\left(U_{2}\left(w_{0}-\Pi+I(W)-W\right)\right)\right) \geq-f_{2}^{\prime}\left(F_{W}(\varepsilon)\right) \int_{0}^{\varepsilon} t U_{2}^{\prime}\left(w_{0}-\Pi-t\right) d F_{W}(t)$

On the other hand, let $h$ be any density in $\partial E_{f_{1}} U_{1}\left(\Pi-I_{\varepsilon}(W)\right)$. By Carlier and Dana [2]lemma 1, $h \geq f_{1}^{\prime}(0)$ a.e.. Hence

$$
E_{f_{1}}\left(U_{1}\left(\Pi-I_{\varepsilon}(W)\right)-E_{f_{1}}\left(U_{1}(\Pi-I(W)) \geq U_{1}^{\prime}(\Pi) f_{1}^{\prime}(0) \int_{0}^{\varepsilon} t d F_{W}(t) .\right.\right.
$$

It follows that from $(25)$ and $(26)$ that

$$
F_{\lambda}\left(I_{\varepsilon}\right)-F_{\lambda}(I) \geq \int_{0}^{\varepsilon} t\left(U_{1}^{\prime}(\Pi) f_{1}^{\prime}(0)-\lambda U_{2}^{\prime}\left(\left(w_{0}-\Pi-t\right) f_{2}^{\prime}\left(F_{W}(\varepsilon)\right) d F_{W}(t)\right.\right.
$$

Since $f_{2}^{\prime}\left(F_{W}(\varepsilon)\right) \rightarrow 0, U_{1}^{\prime}(0)>0$ and $f_{1}^{\prime}(0)>0$, we obtain that $F\left(I_{\varepsilon}\right)>F_{\lambda}(I)$ for $\varepsilon>0$ small enough, hence a contradiction.

If $I^{*}$ is interior, by proposition 3 , the first order conditions are:

$$
f_{1}^{\prime}\left(Z_{1}(\omega)\right) U_{1}^{\prime}\left(x_{1}^{*}(W(\omega))\right)=\lambda f_{2}^{\prime}\left(Z_{2}(\omega)\right) U_{2}^{\prime}\left(x_{2}^{*}(W(\omega))\right)
$$


with $Z_{i}(\omega)=F_{W}(W(\omega))$ for all $\omega$ which does not belong to a level set of $x_{i}^{*}(W)$ with positive measure. It follows in particular from the previous paragraph that $x_{2}^{*}$ is not constant in a neighborhood of 0 . Hence there exists an decreasing sequence $w_{n}$ with limit 0 and such that for almost every $\omega \in\left\{W=w_{n}\right\}, Z_{2}(\omega)=F_{W}\left(w_{n}\right)$. Hence for every $n$, we have:

$$
\lambda f_{2}^{\prime}\left(F_{W}\left(w_{n}\right)\right) U_{2}^{\prime}\left(x_{2}^{*}(W(\omega))\right)=U_{1}^{\prime}\left(x_{1}^{*}\left(w_{n}\right)\right) f_{1}^{\prime}\left(s_{1}(\omega)\right), \forall \omega \in\left\{W=w_{n}\right\}
$$

Passing to the limit, we have a contradiction the left hand side going to 0 while the right hand side being bounded below. Hence $I^{*}$ is not interior in a neighborhood of 0 . In other words, $I(t)=0$ for $t$ small enough

\section{Proof of Proposition 7}

Since $I^{*}$ is Pareto-Optimal, there exists $\lambda \geq 0$ such that $I^{*}$ maximizes

$F_{\lambda}(I):=E_{f_{1}}\left(U_{1}(\Pi-I(W))\right)+\lambda\left((1-\varepsilon) E_{P}\left[U_{2}\left(w_{0}-\Pi+I(W)-W\right)\right]+\varepsilon \min _{\Omega} U_{2}\left(w_{0}-\Pi+I(W)-W\right)\right)$

subject to the constraint $0 \leq I \leq W$. The first order condition $0 \in \partial F_{\lambda}\left(I^{*}\right)$ can be written as the equality of two measures:

$$
h U_{1}^{\prime}\left(\Pi-I^{*}(W)\right) P=\lambda\left(\varepsilon m U_{2}^{\prime}\left(w_{0}-\Pi+I^{*}(b)-b\right)+(1-\varepsilon) U_{2}^{\prime}\left(w_{0}-\Pi+I^{*}(W)-W\right) P+\mu+\nu\right)
$$

where $h \in \operatorname{core} f(P)$ is a strictly positive integrable function, $m$ is supported by $\left\{I^{*}(W)=I^{*}(b)\right\}, \mu$ is supported by $\left\{I^{*}(W)=0\right\}$ and $\nu$ is supported by $\left\{I^{*}(W)=W\right\}$.

Assume by contradiction that $I^{*}(t)-t$ is not constant in any neighbourhood of $b$. Since $I^{*}-\operatorname{Id}$ is non increasing, $I^{*}(t)-t<I^{*}(0)=0$ for $t$ close to $b$. Hence there exists a neighbourhood $\mathcal{V}$ of $b$ such that $\nu$ vanishes on $\left\{I^{*}(W) \in \mathcal{V}\right\}$ and furthermore $m$ is supported by $\{W=b\}$

Let us now prove that similarly $\mu$ vanishes on $I^{*}(W) \in \mathcal{V}$. If not, we would have, since $I^{*}$ is nondecreasing, $I^{*}(t)=0$ for all $t \in[0, b]$. Let us prove then that $I^{*}=0$ cannot be optimal. For $\delta>0$ small enough, consider the contract

$$
I_{\delta}(t):=\max (0, t-b+\delta)
$$

We first have,

$$
\min _{t \in[0, b]} U_{2}\left(w_{0}-\Pi-\mathrm{Id}+I_{\delta}\right)-\min _{t \in[0, b]} U_{2}\left(w_{0}-\Pi-\mathrm{Id}\right)=U_{2}\left(w_{0}-\Pi-b+\delta\right)-U_{2}\left(w_{0}-\Pi-b\right) \geq c_{0} \delta(3
$$

for some constant $c_{0}>0$. Moreover, we have for any $h \in \partial E_{f_{1}}\left(U_{1}\left(\Pi-I_{\delta}(W)\right)\right)$

$$
\begin{aligned}
E_{f_{1}}\left(U_{1}\left(\Pi-I_{\delta}(W)\right)\right)-E_{f_{1}}\left(U_{1}(\Pi)\right) & \geq-\int_{\Omega} I_{\delta}(W) U_{1}^{\prime}\left(\Pi-I_{\delta}(W)\right) h d P \\
& \geq-f_{1}^{\prime}(1) \int_{\Omega} I_{\delta}(W) U_{1}^{\prime}\left(\Pi-I_{\delta}(W)\right) d P \\
& =-f_{1}^{\prime}(1) \int_{b-\delta}^{b} I_{\delta}(t) U_{1}^{\prime}\left(\Pi-I_{\delta}(t)\right) d F_{W} \\
& \geq-c_{1} \delta f_{1}^{\prime}(1)\left(1-F_{W}(b-\delta)\right)
\end{aligned}
$$

for some constant $c_{1}>0$. The second inequality follows from the fact that for every $h \in \operatorname{core} f(P), 0 \leq$ $h(\omega) \leq f_{1}^{\prime}(1)$ (see Carlier \& Dana [2], lemma 1) and the last inequality $0 \leq I_{\delta} \leq \delta$. It follows from (30) and (31) that

$$
F_{\lambda}\left(I_{\delta}\right)-F_{\lambda}(0) \geq \delta\left(c_{0} \varepsilon \lambda-c_{1} f_{1}^{\prime}(1)\left(1-F_{W}(b-\delta)\right)\right)
$$

hence for $\delta$ small enough, $F_{\lambda}\left(I_{\delta}\right)>F_{\lambda}(0)$ which shows that $I^{*}=0$ cannot be optimal and that $\mu$ vanishes for $t$ close to $b$. 
Finally, we may rewrite (29) as

$$
\alpha P=\beta m
$$

for some function $\alpha$ and some constant $\beta>0$ contradicting the assumption that $P$ is non atomic and that $\widetilde{m}$ is concentrated on the set $\{W=b\}$ which has zero measure since $F_{W}^{-1}$ is strictly increasing. Hence $I^{*}(t)-t$ is constant in any neighbourhood of $b$ as was to be proven.

In the case $f_{1}^{\prime}(1)=\infty$, the proof is identical except that it may not be proven that $I^{*}=0$ cannot be optimal

Acknowledgements The authors gratefully acknowledge the support of the ANR, project "Croyances" and of the Fondation du Risque, Chaire Groupama, "Les particuliers face au risque".

\section{References}

[1] Arrow K.J., Uncertainty and the Welfare of Medical Care, American Economic Review, $\mathbf{5 3}$ pp 941-973.

[2] Carlier G., Dana R.A., Core of a convex distortion of a probability on a non atomic space, Journal of Economic Theory, 113, 199-222,(2003).

[3] Chew S.H., Karni E. and Safra E., Risk aversion in the theory of expected utility with Rank dependent probabilities, Journal of Economic Theory, 42, pp.370-381.

[4] Chew S.H., Mao M.H., A Schur Concave Characterization of Risk Aversion for Non Expected Utility Preferences, Journal of Economic Theory, 67, 402-435. (1995)

[5] Dana R.A., A representation result for concave Schur concave functions. To appear in Mathematical Finance. (2005).

[6] Denneberg D., Non-additive Measures and Integral, Kluwer Academic Publishers, Holland, (1994).

[7] Dow, J. and Werlang S., Uncertainty aversion, risk aversion, and the optimal choice of portofolio", Econometrica, 60, pp.197-204, (1992).

[8] Eichberger J. and Kelsey D., E Capacities and the Ellsberg Paradox, Theory and Decision, 46, pp. 107-140, (1992).

[9] Epstein L. and Wang T. , Intertemporal Asset Pricing under Knightian Uncertainty, Econometrica, 62, pp.283-322, (1994).

[10] Hubermann G., Mayers D. and Smith C.W., Optimal insurance policy indemnity schedules, Bell Journal of Economics, 415-426, (1983).

[11] Raviv A., On the Design of an Optimal Insurance Policy", The American Economic Review 69, pp. 84-96, (1979).

[12] Safra Z. and Zilcha I., Efficient sets with and without the expected utility hypothesis Journal of Mathematical Economics, 17, pp. 369-284, (1988)

[13] Winter R. D., Moral Hazard and Insurance Contracts, Contributions to Insurance Economics, Kluwer Academic Publishers, Holland, (1992). 